\title{
1H-NMR based metabolomic profiling of cord blood in gestational hypothyroidism
}

\author{
Chunchao Zhao ${ }^{1 \#}$, Jun Ge ${ }^{1 \#}$, Ruifen Jiao ${ }^{1}, \mathrm{Xia} \mathrm{Li}^{1}$, Yuan $\mathrm{Li}^{1}$, Huili Quan ${ }^{1}$, Tianxiao Yu ${ }^{1}$, Hong Xu${ }^{1}$, \\ Jianguo Li ${ }^{2}$,ing Guo ${ }^{1}$, Wenju Wang ${ }^{1}$
}

${ }^{1}$ Clinical Research Center for Obstetrics and Gynecology, Shijiazhuang Obstetrics and Gynecology Hospital, Shijiazhuang 050000, China; ${ }^{2}$ Department of Systems Biology, Institute of Biomedical Sciences, Shanxi University, Taiyuan 030006, China

Contributions: (I) Conception and design: J Li, C Zhao, W Wang, Q Guo; (II) Administrative support: None; (III) Provision of study materials or patients: C Zhao, J Ge, R Jiao, X Li, Y Li, H Quan, T Yu, H Xu, J Li; (IV) Collection and assembly of data: C Zhao, J Ge, R Jiao, X Li, Y Li, H Quan, T Yu, H Xu, J Li; (V) Data analysis and interpretation: C Zhao, J Ge, R Jiao, X Li, Y Li, H Quan, T Yu, H Xu, J Li; (VI) Manuscript writing: All authors; (VII) Final approval of manuscript: All authors.

\#These authors contributed equally to this work.

Correspondence to: Prof. Wenju Wang; Prof. Qing Guo. Shijiazhuang Obstetrics and Gynecology Hospital, No.206, Zhongshandong Road, Changan District, Shijiazhuang 050000, China. Email: wangwjsj@@126.com; guoqingyz2019@163.com. Dr. Jianguo Li, Key Laboratory of Chemical Biology and Molecular Engineering of Ministry of Education, Shanxi University, No. 92, Wucheng Road, Xiaodian District, Taiyuan 030006, China. Email: lijg@sxu.edu.cn.

Background: Gestational hypothyroidism (GHT) is a common pregnancy-related thyroid disfunction. The adverse outcomes by GHT has been increasingly recognized, leading to more public awareness of the disease. However, comprehensive understanding of the prognosis of GHT has not yet achieved. Metabolomics is a powerful tool in evaluation of disease outcomes, and cord blood represents an excellent candidate for the investigation of gestational outcomes.

Methods: In the present study, we performed 1H-NMR based metabolomics on cord blood of 18 pregnant women with GHT and 18 non hypothyroidism (NHT) control.

Results: The metabolomic profile of GHT was separated with the NHT control. A total of 8 metabolites with altered abundances were observed, among which Creatinine and O-Phosphocholine were elevated and the others were downregulated in GHT. Spearman rank correlation suggested that the eight differential metabolites were correlated with the GHT related thyroid hormones. Pathway analysis of the differential metabolites indicated that two metabolic pathways were significantly altered in GHT (adjusted P<0.05), including tyrosine metabolism, phenylalanine, tyrosine and tryptophan biosynthesis. Enrichment analysis of the differential metabolites against disease-associated metabolite sets suggested that GHT was associated with disease risks of non-insulin dependent diabetes mellitus, isovaleric acidemia, and methylmalonic aciduria.

Conclusions: The results of this study revealed GHT associated metabolic changes in cord blood, providing insights into the metabolic intermediates between GHT and its related disease risks.

Keywords: Gestational hypothyroidism (GHT); metabolomics; prognosis; cord blood; 1H-NMR

Submitted Nov 27, 2019. Accepted for publication Feb 05, 2020.

doi: 10.21037/atm.2020.03.91

View this article at: http://dx.doi.org/10.21037/atm.2020.03.91 


\section{Introduction}

Gestational hypothyroidism (GHT), a common endocrinopathy, exerts various adverse outcomes to mother and the fetus (1). GHT has been associated with preeclampsia, anemia, abruption placenta, miscarriage, and postpartum hemorrhage (2). Besides the adverse effects during pregnancy, GHT also does harm to future health of mother and long-term development of the offspring $(3,4)$. Comprehensive evaluations of the GHT prognosis is helpful in promoting public awareness of GHT (5), and is instrumental in guiding standard treatment of GHT (6).

Metabolomics, defined as the comprehensive analysis of metabolites in a biological specimen, is a powerful approach in prognosis of diseases (7-9). Pathophysiological alterations will inevitably lead to changes in the abundances of specific metabolites (10). Metabolomics affords a comprehensive coverage of biological processes and metabolic pathways through profiling of larger number of metabolites than the current clinical laboratory techniques (11), which hold promise to serve as an essential objective lens in the molecular microscope for precision medicine (11). Metabolomics offers a promising tool for the discovery of biomarkers that could be applied in disease diagnosis $(9,12,13)$, and in evaluation of therapeutic response to a therapy (14-17).

Although metabolomics has been widely applied in studying gestational diabetes mellitus (18) and gestational hypertension (19), application of metabolomics on GHT is rare. For comprehensive understanding of the adverse outcomes of GHT, it is necessary to evaluate the influences of GHT by multifarious approaches (20). In the present study, we performed $1 \mathrm{H}-\mathrm{NMR}$ based metabolomics on cord blood to assess the metabolic consequences of GHT.

\section{Methods}

\section{Study participants}

A cohort of 36 pregnant women entered the Fourth Hospital of Shijiazhuang (Hebei province, PR China) during 2018 were enrolled in this study, including 18 women with GHT, and 18 non hypothyroidism (NHT) control. GHT was diagnosed according to the 2017 Guidelines of the American Thyroid Association for the Diagnosis and Management of Thyroid Disease During Pregnancy and Postpartum (21). Women who met any of the following criteria were defined as GHT: (I) ThyroidStimulating Hormone (TSH) level $>4.2 \mathrm{mIU} / \mathrm{L}$; (II) Free Tri- iodothyonine (FT3) level $<3.1 \mathrm{pmol} / \mathrm{L}$; (III) Free Thyroxine (FT4) level $<12$ pmol/L. Women who simultaneously met the following criteria were defined as NHT: (I) TSH level ranges from 0.27 to $4.2 \mathrm{mIU} / \mathrm{L}$; (II) FT3 level ranges from 3.1 to $6.8 \mathrm{pmol} / \mathrm{L}$; (III) FT4 level ranges from 12 to $22 \mathrm{pmol} / \mathrm{L}$. Only the pregnant women who met the above GHT criteria both at $11^{\text {th }}-14^{\text {th }}$ gestational week and after the parturition were included into GHT group. Women with known pre-existing thyropathy or autoimmune disease, goiters, thyroid peroxidase antibody were excluded. The study was conducted according to the guidelines in the Declaration of Helsinki, and approved by the Ethics Committee of the Fourth Hospital of Shijiazhuang.

\section{Demographic data and sample collection}

Demographic data of the enrolled pregnant women were obtained by interview at the $11^{\text {th }}-14^{\text {th }}$ gestational week, including age, height, bodyweight (before pregnancy and at the interview). Clinical information, including type of delivery and gestational age was collected after the parturition. Cord blood was collected during the parturition. EDTA anticoagulant plasma was extracted for metabolomic profiling by centrifuge at $4{ }^{\circ} \mathrm{C}, 3,000 \mathrm{rpm}$ for $15 \mathrm{~min}$, aliquoted and stored in a deep freezer at $-80^{\circ} \mathrm{C}$.

\section{Measurements of TSH, FT3 and FT4}

TSH, FT3 and FT4 was measured from fasting serum by a Cobas 6000 (Roche Diagnostics, Basil Switzerland) analyzers utilizing electrochemiluminescence (ECL) technology. The analyzer was calibrated using both pathological and normal controls according to the manufacturer's instructions. Method calibrations were done on the analyzer once a month to reduce systemic bias.

\section{H-NMR based metabolomics}

Plasma sample preparation for $1 \mathrm{H}-\mathrm{NMR}$ spectral profiling was performed according to a previously reported procedure with slight modifications (22). Briefly, an aliquot of $450 \mu \mathrm{L}$ plasma was thawed on ice and mixed with 900 $\mathrm{\mu l}$ methanol, vortexed for $2 \mathrm{~min}$ then centrifuged at $4{ }^{\circ} \mathrm{C}$, 13,000 rpm for $20 \mathrm{~min}$ to pellet proteins. The supernatant was dried completely by a vacuum dryer (Concentrator Plus, Eppendorf, Germany). The dried sample was reconstituted in $600 \mu \mathrm{L}$ phosphate buffer solution in $\mathrm{D}_{2} \mathrm{O}$ $\left(0.2 \mathrm{M} \mathrm{Na}_{2} \mathrm{HPO}_{4} / \mathrm{NaH}_{2} \mathrm{PO}_{4}, \mathrm{pD}=7.4\right.$, containing $10 \mathrm{mM}$ 
TSP as internal standard). The sample was then centrifuged at $4{ }^{\circ} \mathrm{C}, 13,000 \mathrm{rpm}$ for $20 \mathrm{~min}$ to eliminate the precipitates, and $550 \mu \mathrm{L}$ of the supernatant was transferred into a $5 \mathrm{~mm}$ NMR tube for NMR spectrometry by a Bruker $600 \mathrm{MHz}$ AVANCE III NMR spectrometer (Bruker BioSpin, Germany). The NMR spectrum was acquired using Carr-Purcell-Merboom-Gill (CPMG) pulse sequences with the following parameters: spectral width $12,019.2 \mathrm{~Hz}$, spectral size 65,536 points, pulse width $14 \mu \mathrm{s}$, relaxation delay $1.0 \mathrm{~s}$, scan number 64. MestReNova (v8.0.1, Mestrelab Research, Santiago de Compostella, Spain) was used for spectra processing. The phase and baseline were corrected manually and the chemical shift of TSP was calibrated at $0.00 \mathrm{ppm}$. The spectra region of $\delta 0.11$ to $\delta 9.12$ were segmented at $0.01 \mathrm{ppm}$ width into $1 \mathrm{H}-\mathrm{NMR}$ features after exclusion of the region of residual water $(\delta 4.75-\delta 5.08)$. The obtained NMR data was normalized to the total sum of spectra before further analysis.

\section{Multivariate analysis}

MetaboAnalyst (v4.0, http://www.metaboanalyst.ca) was applied for multivariate analysis of the metabolomic data (23). Principal component analysis (PCA) was applied for an overview of the metabolomic data. Partial-Least Squares Discrimination Analysis (PLS-DA) was applied to select the 1H-NMR features with discriminative power between the GHT group and the NHT group. A 200-cycle permutation test was used to evaluate the PLS-DA model. The fitting validity and predictive ability of the PLSDA model were assessed by the parameters of multiple correlation coefficient (R2) and the cross-validated R2 (Q2). $1 \mathrm{H}-\mathrm{NMR}$ features with discriminative power were validated by Wilcoxon Rank Test and EBAM [Empirical Bayesian Analysis of Microarray (and Metabolites)] implemented in MetaboAnalyst. Metabolites were characterized from the 1H-NMR features with discriminative power by searching against the human metabolome database (HMDB, http:// www.hmdb.ca) and previously published literatures with chemical shift, coupling constant, and peak type. Metabolic pathway enrichment was performed by the enrichment analysis module of the MetaboAnalyst web portal.

\section{Results}

\section{Data pre-processing and quality control of the plasma metabolome}

To avoid the influences of water and EDTA (as anticoagulant), we deleted the water and EDTA peaks before further investigations (Figure S1). No missing value was detected by a data integrity check of the metabolomic data. The metabolic data was then subjected to a data preprocessing procedure including sample normalization by sum, $\log$ transformation (for adjustment of positive skewness of the metabolomic data) and scaling (UV scaling for PCA and Pareto scaling for PLS-DA). A better distribution of the metabolomic data was observed after the pre-processing procedure (Figure S2). To monitor the robustness and reproducibility of the metabolomic profiling, quality controls (QCs) were applied by pooling equal aliquots of each plasma sample, and were analyzed after every ten clinical samples. Quality control of the metabolomic data was performed by PCA on the QCs and clinical samples. All of the QCs were clustered together in the sample space of PCA (Figure S3), indicating a good reproducibility of the metabolic profiling.

\section{Multivariate analysis suggests notable variations occurred in the cord blood metabolome of GHT}

To primarily evaluate the natural separation of the cord blood plasma metabolome among the 36 enrolled pregnant women, PCA was performed on the obtained $1 \mathrm{H}-\mathrm{NMR}$ spectra (Figure 1A). The first two principle components (PCs) could explain $47.3 \%$ of the overall variances $(30.3 \%$ and $17 \%$ for PC1 and PC2, respectively). The plasma metabolome of GHT exhibited a clear separation with those of the NHT group, indicating a natural separation occurred in the cord blood metabolome of the two groups.

To better discriminate the metabolome between the GHT group and the NHT group, and to explore the metabolites responsible for the variations, PLS-DA was performed on the cord blood metabolome. Multiple correlation coefficient $(\mathrm{R} 2=0.66)$ and the cross-validated $\mathrm{R} 2(\mathrm{Q} 2=0.41)$ suggested good fitting validity and predictive 
A

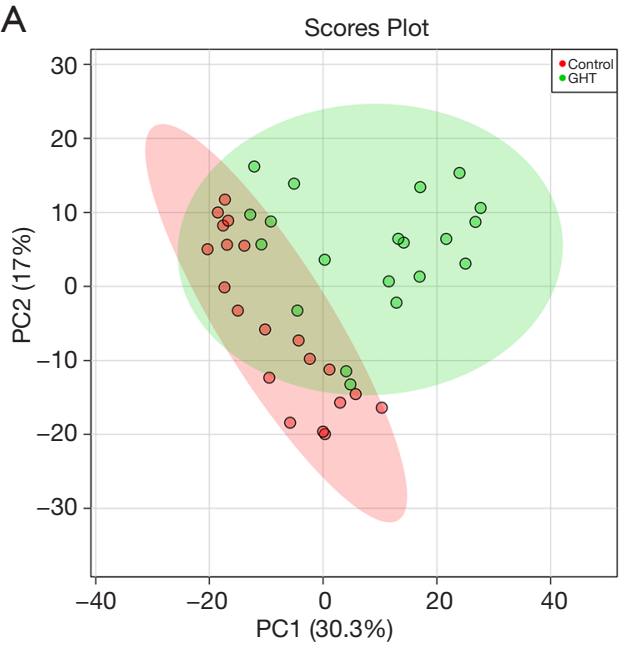

B

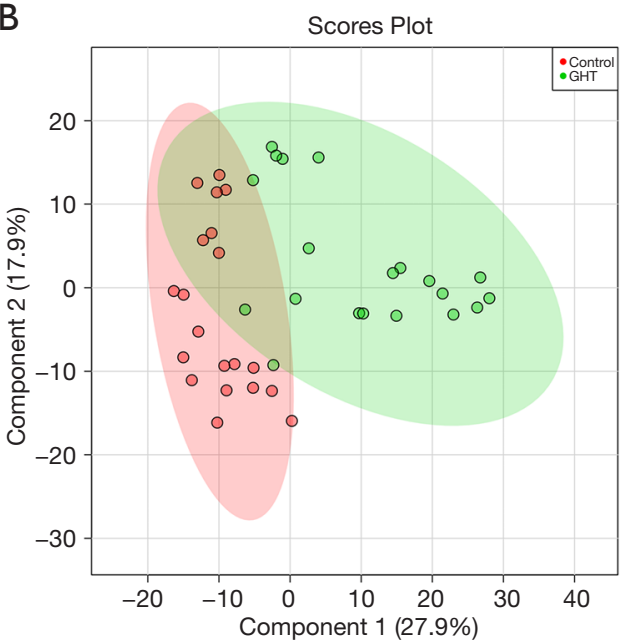

Figure 1 PCA and PLS-DA on the cord blood plasma metabolome between women with GHT and the NHT control. (A) Scores plot of PCA based on the first two principle components (PCs), which explain $47.3 \%$ of the total variances $30.3 \%$ and $17 \%$ for PC1 and PC2, respectively); (B) scores plot of PLS-DA based on component 1 and component 2, which explain $45.8 \%$ of the total variances $(27.9 \%$ and $17.9 \%$ for component 1 and component 2, respectively). Red oval represents the $95 \%$ CI of scores calculated from the NHT controls. Green oval represents the 95\% CI of scores calculated from the GHT samples. PCA, Principle Component Analysis; PLS-DA, Projection to Latent Structures-Discrimination Analysis; GHT, gestational hypothyroidism; NHT, non-hypothyroidism.

ability of the PLS-DA model. A 200-cycle permutation test (Figure S4) was performed to evaluate the statistical significance of the obtained PLS-DA model, the result of which indicated that the observed between-group differences are assumed to be statistically significant $(\mathrm{P}=0.009)$. From the score plot of PLS-DA (Figure 1B), the GHT group was clustered and clearly separated with the NHT group, suggesting significant variations occurred in the circulating metabolic profiles of pregnant women with GHT.

\section{Identification of metabolites with altered abundances in cord blood of GHT}

The metabolites with significant altered abundances between GHT and the NHT control were identified from the segmented $1 \mathrm{H}-\mathrm{NMR}$ features obtained from 1H-NMR spectra (http://cdn.amegroups.cn/static/ application/8dc7cd077693e1cedf783970bc6c4cda/ atm.2020.03.91-1.pdf). The between-group differential $1 \mathrm{H}-\mathrm{NMR}$ features should simultaneously meet the following criteria: significant changed relative abundance (a peak area divided by the total peak area of a sample) with adjusted $\mathrm{P}<0.05$ in a Wilcoxon Rank Test (Figure $2 A$, Table S1); the calculated posterior larger than 0.9 and the $\mathrm{z}$ value lager than 2 in EBAM (Figure $2 B$, Table S2); Variable Importance for the Projection (VIP) greater than 1 in the PLS-DA model. A total of 19 1H-NMR features with altered abundances between GHT and NHT were obtained, among which 8 metabolites were assigned (structurally identified) against the database of HMDB and the previously published literatures (Table 1). The absolute abundances (Figure 3) of the differential metabolites (calculated by the abundance of TSP multiplied by the quotient of the peak area of the metabolite and the peak area of TSP) suggested that Creatinine (adjusted $\mathrm{P}<0.01$, Figure $3 A$ ) and $\mathrm{O}-\mathrm{Phosphocholine} \mathrm{(adjusted} \mathrm{P}<0.01$, Figure $3 B$ ) were elevated in GHT group, while a panel of 6 metabolites were decreased in GHT group including Tyrosine (adjusted $\mathrm{P}<0.01$, Figure $3 C$ ), Citric acid (adjusted $\mathrm{P}<0.01$, Figure 3D), 3-Methyl-2-Oxovaleric acid (adjusted $\mathrm{P}<0.01$, Figure $3 E$ ), Sarcosine (adjusted $\mathrm{P}<0.05$, Figure $3 F$ ), Carnitine (adjusted $\mathrm{P}<0.05$, Figure $3 G$ ), L- $\alpha$-Aminobutyric acid (adjusted $\mathrm{P}<0.05$, Figure $3 H$ ).

\section{The differential cord blood metabolites were correlated with the clinical indices of GHT}

To investigate the relationship between the differential metabolites and GHT associated clinical indices (Table S3), 
A

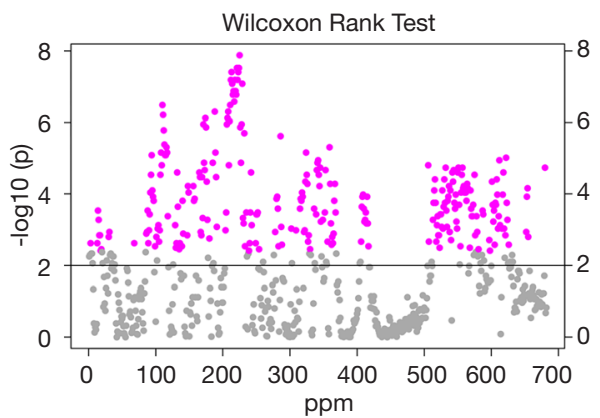

B

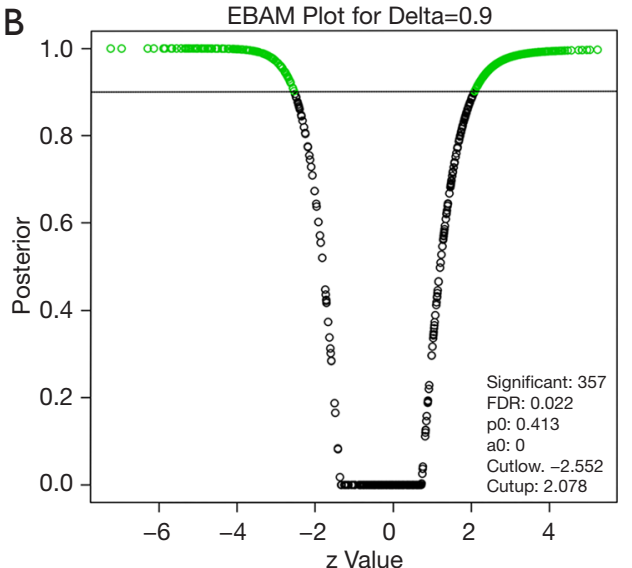

Figure 2 Selection of the 1H-NMR features with altered abundances in cord blood plasma metabolome of women with GHT. (A) Wilcoxon rank test to the whole 682 features extracted from the $1 \mathrm{H}-\mathrm{NMR}$ spectrometry on the cord blood plasma of the enrolled pregnant women. A total of 272 features with adjusted $\mathrm{P}<0.01$ were selected (pink dots); (B) EBAM [Empirical Bayesian Analysis of Microarray (and Metabolites)] on the whole 682 features. A total of 357 features with posterior greater than 0.9 were selected (green circles). GHT, gestational hypothyroidism.

Table 1 Cord blood metabolites with altered abundances between GHT and the NHT control

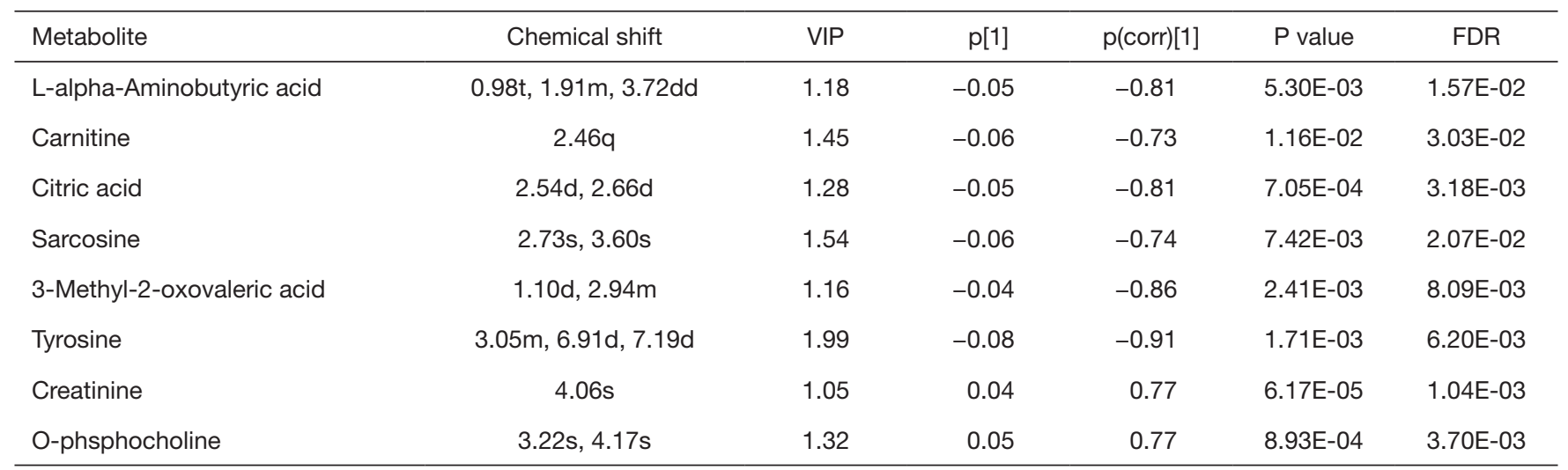

GHT, gestational hypothyroidism; NHT, non-hypothyroidism; VIP, variable importance in the projection; FDR, false discovery rate; s, singlepeak; d, doublet-peak; t, triplet-peak; q, quartet-peak; m, multiplet-peak; p[1], covariance coefficient calculated with Principle Component 1 of the selected OPLS-DA model; p(corr)[1], correlation coefficient calculated with Principle Component 1 of the selected OPLS-DA model.

spearman rank correlation was performed (Figure 4A). Among the thyroid hormones, TSH was negatively correlated with L- $\alpha$-Aminobutyric acid $(\rho=-0.32$, $\mathrm{P}=7.01 \mathrm{E}-04)$, Carnitine $(\rho=-0.30, \mathrm{P}=5.01 \mathrm{E}-04)$, Citric acid $(\rho=-0.30, P=3.76 \mathrm{E}-04)$, Sarcosine $(\rho=-0.35, P=1.18 \mathrm{E}-04)$, 3 -Methyl-2-Oxovaleric acid $(\rho=-0.28, P=4.67 \mathrm{E}-04)$, and Tyrosine $(\rho=-0.30, P=4.65 \mathrm{E}-04)$, positively correlated with Creatinine $(\rho=0.48, P=3.18 \mathrm{E}-04)$, and $\mathrm{O}-\mathrm{Phosphocholine}$ $(\rho=0.31, P=4.08 \mathrm{E}-03)$. The relationship between TSH and the differential metabolites were similar with those of FT3, but in contrary to those of FT4 (Figure 4A). Delta BMI (the difference of Body Mass Index (BMI) between the sampling time and before pregnancy) was negatively correlated with L- $\alpha$-Aminobutyric acid $(\rho=-0.20, P=2.94 E-02)$, Carnitine $(\rho=-0.08, P=4.42 E-02)$. No significant correlation $(P<0.05)$ was observed between the differential metabolites and abdomen circumference, fundal height, respectively. Among the 8 plasma metabolites, Creatinine and O-Phosphocholine were positively correlated with each other $(\rho=0.67$, $\mathrm{P}=3.47 \mathrm{E}-07)$, and negatively correlated with the other 6 

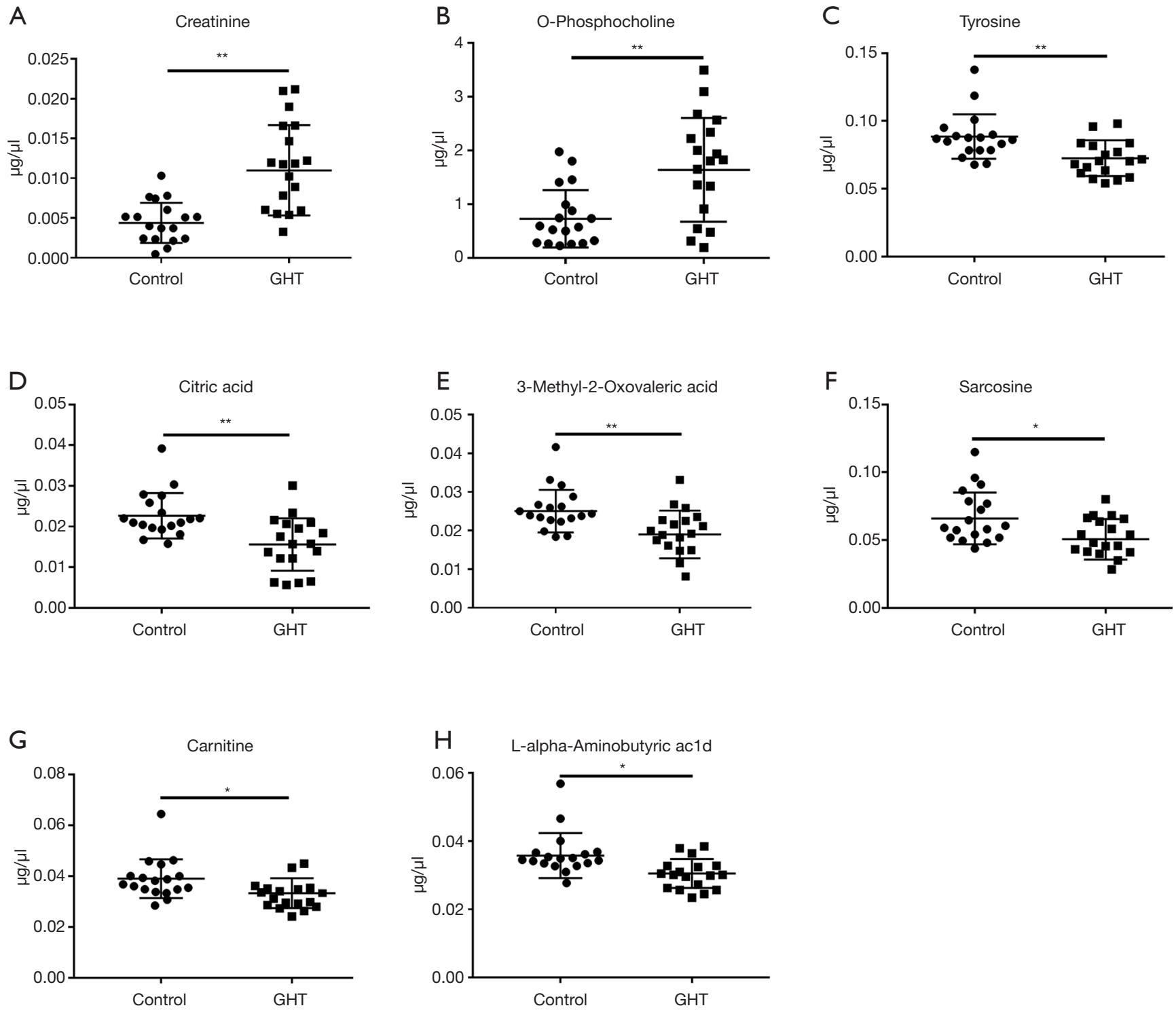

Figure 3 Absolute abundance of the metabolites with differential abundances in cord blood between women with GHT and the NHT control. The absolute abundance of each metabolite was calculated by the abundance of TSP multiplied by the quotient of the peak area of the metabolite and the peak area of TSP. Nonparametric test was applied to evaluate the between-group statistical significance. Multiple comparison adjustment was performed by False discovery rate. * $\mathrm{P}<0.05$; **, $\mathrm{P}<0.01$. GHT, gestational hypothyroidism; NHT, nonhypothyroidism.

plasma metabolites (Figure 4A) These results suggested that cord blood plasma metabolites may contribute to changes in GHT related clinical indices.

\section{Tyrosine metabolism, phenylalanine, tyrosine and tryptophan biosynthesis were altered in GHT}

Because the GHT associated differential cord blood plasma metabolites exhibited significant correlations among each other (Figure 4A), we speculated that shared metabolic pathways existed. For further biological interpretation of the differential metabolites between GHT and the NHT control, metabolic pathway analysis was performed by the pathway analysis module implemented in the MetaboAnalyst web portal (Figure 4B). Tyrosine metabolism (pathway impact $=0.14$, adjusted $\mathrm{P}=2.92 \mathrm{E}-03$ ) and phenylalanine, 
A

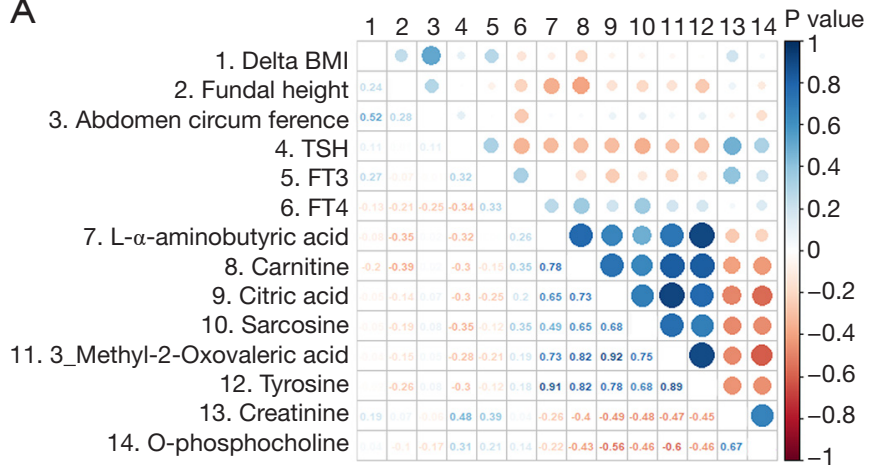

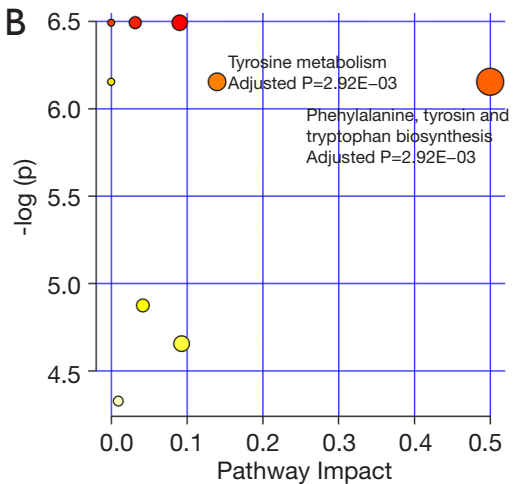

Figure 4 Spearman correlation and pathway analysis of the metabolites with altered abundances in cord blood of women with GHT. (A) Spearman rank correlations among the clinical indices of GHT and the metabolites with altered abundances in cord blood of women with GHT. The color square represents he rho values of spearman's coefficient, corresponding to the color bar on the right. The bigger the color square, the higher the absolute rho value. The numerical rho values are listed in Table S3. Star represents the statistical significance of spearman rank correlation; (B) significant pathway of the metabolites with differential abundances between GHT and NHT. Pathway analysis was performed by the pathway analysis module of MetaboAnalyst web portal against the KEGG library. Hypergeometric test was applied for over representation analysis. Relative-betweenness centrality was used for pathway topology analysis. Only pathways with adjusted P values lower than 0.05 were labelled. GHT, gestational hypothyroidism; NHT, non-hypothyroidism.

Table 2 Enrichment analysis of the differential metabolites against disease-associated metabolite sets

\begin{tabular}{lccccc}
\hline Metabolite set & Total & Hits & Hit metabolite & P value & Adjusted P value \\
\hline Metabolites affected by gender & 9 & 2 & Tyrosine, creatinine & $1.11 \mathrm{E}-05$ & $4.15 \mathrm{E}-04$ \\
Diabetes mellitus (non-insulin dependent) & 19 & 2 & Citric acid, creatinine & $3.69 \mathrm{E}-05$ & $4.15 \mathrm{E}-04$ \\
Isovaleric acidemia & 9 & 2 & Creatinine, carnitine & $4.05 \mathrm{E}-05$ & $4.15 \mathrm{E}-04$ \\
Methylmalonic aciduria & 8 & 2 & Creatinine, carnitine & $4.05 \mathrm{E}-05$ & $4.15 \mathrm{E}-04$ \\
\hline
\end{tabular}

tyrosine and tryptophan biosynthesis (pathway impact $=0.50$, adjusted $\mathrm{P}=2.92 \mathrm{E}-03$ ) were significantly altered in GHT. Other significantly altered (adjusted $\mathrm{P}<0.05$ ) metabolic pathways with lower pathway impact $(0<$ pathway impact $<0.1)$ were also observed, including citrate cycle, glyoxylate and dicarboxylate metabolism, cysteine and methionine metabolism, glycine, serine and threonine metabolism, glycerophospholipid metabolism. These results suggested that alterations in metabolic pathways occurred in cord blood of GHT, indicating potential disease risks.

\section{Enrichment analysis of the differential metabolites against disease-associated metabolite sets}

To evaluate the GHT associated disease risks, we performed enrichment analysis of the differential metabolites against disease-associated metabolite sets by the enrichment analysis module of MetaboAnalyst web portal. Only the disease-associated metabolite sets hit by no less than 2 differential metabolites with adjusted $\mathrm{P}<0.05$ were listed (Table 2). Among the enriched disease-associated metabolite sets, metabolites affected by gender was hit by Tyrosine and Creatinine, Diabetes mellitus (non-insulin dependent) was hit by Citric acid, and Creatinine, Isovaleric academia was hit by Creatinine and Carnitine, methylmalonic aciduria was hit by Creatinine and Carnitine. These results illustrated the metabolic intermediates between GHT and GHT-associated disease risks, providing clues in further investigation of the prognosis of GHT.

\section{Discussion}

GHT is a common pregnancy-related thyroid dysfunction, even mild hypothyroidism may result in adverse gestational 
outcomes. However, little is known about the intermediates between hypothyroidism and its adverse gestational outcomes. While metabolomics is a powerful tool to decipher disease outcomes, and cord blood serves as an excellent candidate to investigate the gestational outcomes, we performed 1H-NMR based metabolomics on cord blood to reveal GHT related metabolic changes, and to bring insight into the correlations between these changes and the clinical manifestations of GHT.

Hypothyroidism is a well-recognized risk factor for insulin resistance, which disrupt hormonal balances and alter the metabolism of lipids, protein and carbohydrate. A panel of 8 plasma metabolites with altered abundances in GHT was observed in the present study, suggesting that GHT is a risk factor of metabolic disorders. In consistence with the findings of this study, a good correlation has been established between thyroid status and the plasma concentrations of several amino acids, including $\alpha$-aminobutyric acid, glycine, glutamic acid and alanine (24). The plasma amino acid concentrations in experimental rats were significantly increased by feeding with desiccated thyroid, and were remarkably decreased after thyroidectomy. The kinetic parameters of electrogenic transfer for sarcosine was significantly altered under hypothyroidism (25). Tyrosine, as a precursor of thyroid hormones, in combination with iodine and the follicular cells, synthesize and secrete thyroid hormone. Thyroid hormones modulate carnitine palmitoyl transferase $1 \alpha$ and acyl-CoA oxidase. The effect of GHT on L- $\alpha$-Aminobutyric acid was rarely reported, although the concentration of GABA was decreased in hippocampus of adult male offspring of mice under mild perinatal hypothyroidism (26). The effects of altered maternal thyroid function on the fetal and neonatal myocardial phospholipid have been documented. Offspring born to hypothyroid rather than hyperthyroid mother has decreased total phospholipids, both at fetal and neonatal stages. Maternal thyroid seems to play an essential role in the regulations of phospholipid metabolism in fetal and neonatal hearts (27). The serum Creatinine is attributable to the hormonal correction of hypothyroidism (28). The plasma Citrate (the salt form of Citric acid) was decreased during treatment of primary hypothyroidism with triiodothyronine (29).

Besides the altered abundances of individual metabolites, two disturbed metabolism pathways were also observed in GHT in the present study, including tyrosine metabolism, phenylalanine, tyrosine and tryptophan biosynthesis. Tyrosine is a critical precursor of thyroid hormone. The thyroid gland combined iodine and tyrosine to make thyroid hormones. Tyrosine metabolism disorder was reported to be a risk factor of insulin resistance and gestational diabetes (30). Although the correlation between GHT and phenylalanine, tyrosine and tryptophan biosynthesis was rarely reported, the aromatic amino acids phenylalanine and tryptophan were found to inhibit the uptake of FT3 (31), illustrating a critical role of this metabolic pathway to GHT. The results of this study, combined with the previous reports, suggested that GHT is associated with metabolic disorders in the circulating system.

Metabolic disorders are risk factors of various diseases, including obesity, diabetes, and even cancer, mood disorders. Metabolomics provides a powerful tool for investigating the prognosis of gestation. In the present study, three GHT related disease risks were predicted, including diabetes mellitus, isovaleric academia and methylmalonic aciduria. In accordance with the findings of this study, hypothyroidism was reported to be a risk factor for diabetes mellitus (32), while TSH level was associated with and increased risk of GDM (33). Isovaleric academia is an inborn error of metabolism, characterized by a deficiency of isovaleryl-CoA dehydrogenase, leading to accumulation of isovalerylcarnitine or isovaleryglycine (34). Methylmalonic aciduria is another inborn error of metabolism, characterized by a deficiency of the enzyme methylmalonyl-CoA mutase (35). Pregnancy is believed to exert impact on inborn errors of metabolism (36), and thyroid dysfunction was associated with a series of inborn errors of metabolism (37). Supported by the previous reports, the GHT enriched metabolite-sets observed in this study provide insights into the connections between GHT and its related disease risks.

As a shortcoming of the present study, EDTA was used as the anticoagulant for $1 \mathrm{H}-\mathrm{NMR}$ spectrometry. EDTA could be detected by NMR spectrometry, and its peaks in the NMR spectra vary depending on the type and concentration of ions present in the sample. We thus deleted the EDTA peaks from all of the obtained NMR spectra before multivariate analysis to avoid its influence. Metabolite confirmation is critical to support the findings of untargeted metabolomics. Further investigations are recommended to confirm the existence and to explore the roles of the GHT associated cord blood metabolites observed in the present study. 


\section{Conclusions}

In conclusion, we performed $1 \mathrm{H}-\mathrm{NMR}$ based metabolomics on cord blood to reveal metabolic changes and related disease risks of GHT. A panel of 8 metabolites with altered abundances in GHT was observed. Three disease risks were predicted by the metabolites with altered abundances, including non-insulin dependent diabetes mellitus, isovaleric academia and methylmalonic aciduria. The results of this study provide insights into the metabolic intermediates between GHT and its related disease risks.

\section{Acknowledgments}

We thank Dr. Jinping Jia from Scientific Instrument Center of Shanxi University for the critical comments on this study. Funding: This work was supported by the National Natural Science Foundation of China (Grant no. 81301441), and Shanxi Soft Science Research Project (Grant no. 2018041031-2).

\section{Footnote}

Conflicts of Interest: The authors have no conflicts of interest to declare.

Ethical Statement: The authors are accountable for all aspects of the work in ensuring that questions related to the accuracy or integrity of any part of the work are appropriately investigated and resolved. The study was conducted according to the guidelines in the Declaration of Helsinki, and approved by the Ethics Committee of the Fourth Hospital of Shijiazhuang.

Open Access Statement: This is an Open Access article distributed in accordance with the Creative Commons Attribution-NonCommercial-NoDerivs 4.0 International License (CC BY-NC-ND 4.0), which permits the noncommercial replication and distribution of the article with the strict proviso that no changes or edits are made and the original work is properly cited (including links to both the formal publication through the relevant DOI and the license). See: https://creativecommons.org/licenses/by-nc-nd/4.0/.

\section{References}

1. Burman KD. Thyroid function: Gestational hypothyroidism--a need for universal screening? Nat Rev
Endocrinol 2012;8:260-1.

2. Taylor PN, Lazarus JH. Hypothyroidism in Pregnancy. Endocrinol Metab Clin North Am 2019;48:547-56.

3. Pearce EN. Thyroid disorders during pregnancy and postpartum. Best Pract Res Clin Obstet Gynaecol 2015;29:700-6.

4. Smith A, Eccles-Smith J, D'Emden M, et al. Thyroid disorders in pregnancy and postpartum. Aust Prescr 2017;40:214-9.

5. Villagelin D, Comarella AP, Tiago DB, et al. Management of gestational hypothyroidism: results of a Brazilian survey. Arch Endocrinol Metab 2016;60:16-20.

6. Donnay S, Fajardo C, Fernandez-Garcia JC, et al. Diagnosis, treatment, and management of gestational hypothyroidism. The TIROGEST study. Endocrinol Diabetes Nutr 2020;67:36-42.

7. Banoei MM, Vogel HJ, Weljie AM, et al. Plasma metabolomics for the diagnosis and prognosis of H1N1 influenza pneumonia. Crit Care 2017;21:97.

8. Duffy FJ, Weiner J, 3rd, Hansen S, et al. Immunometabolic Signatures Predict Risk of Progression to Active Tuberculosis and Disease Outcome. Front Immunol 2019;10:527.

9. Priori R, Scrivo R, Brandt J, et al. Metabolomics in rheumatic diseases: the potential of an emerging methodology for improved patient diagnosis, prognosis, and treatment efficacy. Autoimmun Rev 2013;12:1022-30.

10. Pey J, Tobalina L, de Cisneros JP, et al. A network-based approach for predicting key enzymes explaining metabolite abundance alterations in a disease phenotype. BMC Syst Biol 2013;7:62.

11. Clish CB. Metabolomics: an emerging but powerful tool for precision medicine. Cold Spring Harb Mol Case Stud 2015;1:a000588.

12. Viswan A, Singh C, Rai RK, et al. Metabolomics based predictive biomarker model of ARDS: A systemic measure of clinical hypoxemia. PLoS One 2017;12:e0187545.

13. Wang X, Zhang A, Sun H. Power of metabolomics in diagnosis and biomarker discovery of hepatocellular carcinoma. Hepatology 2013;57:2072-7.

14. Lanznaster D, de Assis DR, Corcia P, et al. Metabolomics Biomarkers: A Strategy Toward Therapeutics Improvement in ALS. Front Neurol 2018;9:1126.

15. Mikami T, Aoki M, Kimura T. The application of mass spectrometry to proteomics and metabolomics in biomarker discovery and drug development. Curr Mol Pharmacol 2012;5:301-16.

16. Guo W, Tan HY, Wang N, et al. Deciphering 
hepatocellular carcinoma through metabolomics: from biomarker discovery to therapy evaluation. Cancer Manag Res 2018;10:715-34.

17. Patel S, Ahmed S. Emerging field of metabolomics: big promise for cancer biomarker identification and drug discovery. J Pharm Biomed Anal 2015;107:63-74.

18. Bentley-Lewis R, Huynh J, Xiong G, et al. Metabolomic profiling in the prediction of gestational diabetes mellitus. Diabetologia 2015;58:1329-32.

19. Austdal M, Tangeras LH, Skrastad RB, et al. First Trimester Urine and Serum Metabolomics for Prediction of Preeclampsia and Gestational Hypertension: A Prospective Screening Study. Int J Mol Sci 2015;16:21520-38.

20. Peixoto AB, Caldas TM, Santos RO, et al. The impact of gestational diabetes and hypothyroidism on the thirdtrimester ultrasound parameters and in adverse perinatal outcomes: a retrospective cohort study. J Matern Fetal Neonatal Med 2016;29:3416-20.

21. Alexander EK, Pearce EN, Brent GA, et al. 2017 Guidelines of the American Thyroid Association for the Diagnosis and Management of Thyroid Disease During Pregnancy and the Postpartum. Thyroid 2017;27:315-89.

22. Liu CC, Wu YF, Feng GM, et al. Plasma-metabolitebiomarkers for the therapeutic response in depressed patients by the traditional Chinese medicine formula Xiaoyaosan: A (1)H NMR-based metabolomics approach. J Affect Disord 2015;185:156-63.

23. Chong J, Soufan O, Li C, et al. MetaboAnalyst 4.0: towards more transparent and integrative metabolomics analysis. Nucleic Acids Res 2018;46:W486-94.

24. Ness GC, Takahashi T, Lee YP. Thyroid hormones on amino acid and protein metabolism. I. Concentration and composition of free amino acids in blood plasma of the rat. Endocrinology 1969;85:1166-71.

25. Syme G, Levin RJ. The effects of hypothyroidism and fasting on electrogenic amino acid transfer: possible evidence for multiple neutral amino acid carrier systems in rat jejunum. Biochim Biophys Acta 1977;464:620-8.

26. Amano I, Takatsuru Y, Khairinisa MA, et al. Effects of

Cite this article as: Zhao C, Ge J, Jiao R, Li X, Li Y, Quan H, Yu T, Xu H, Li J, Guo Q, Wang W. 1H-NMR based metabolomic profiling of cord blood in gestational hypothyroidism. Ann Transl Med 2020;8(6):296. doi: 10.21037/ atm.2020.03.91
Mild Perinatal Hypothyroidism on Cognitive Function of Adult Male Offspring. Endocrinology 2018;159:1910-21.

27. Kumar R, Chaudhuri BN. Altered maternal thyroid function: fetal and neonatal heart cholesterol and phospholipids. Indian J Physiol Pharmacol 1993;37:176-82.

28. Petkov Stoyanov V, Martin Navarro JA, Merida Herrero $\mathrm{E}$, et al. Decrease in renal function associated with hypothyroidism. Nefrologia 2010;30:378-80.

29. Cohen RD. Effect on the plasma citrate of treatment of primary hypothyroidism with triiodothyronine. Nature 1962;196:486-7.

30. Park S, Park JY, Lee JH, et al. Plasma levels of lysine, tyrosine, and valine during pregnancy are independent risk factors of insulin resistance and gestational diabetes. Metab Syndr Relat Disord 2015;13:64-70.

31. Prasad PD, Leibach FH, Mahesh VB, et al. Relationship Between Thyroid-Hormone Transport and Neutral Amino-Acid-Transport in Jar Human Choriocarcinoma Cells. Endocrinology 1994;134:574-81.

32. Gronich N, Deftereos SN, Lavi I, et al. Hypothyroidism is a Risk Factor for New-Onset Diabetes: A Cohort Study. Diabetes Care 2015;38:1657-64.

33. Ying H, Tang YP, Bao YR, et al. Maternal TSH level and TPOAb status in early pregnancy and their relationship to the risk of gestational diabetes mellitus. Endocrine 2016;54:742-50.

34. Chinen Y, Nakamura S, Tamashiro K, et al. Isovaleric acidemia: Therapeutic response to supplementation with glycine, L-carnitine, or both in combination and a 10-year follow-up case study. Molecular Genetics and Metabolism Reports 2017;11:2-5.

35. Baumgartner MR, Horster F, Dionisi-Vici C, et al. Proposed guidelines for the diagnosis and management of methylmalonic and propionic acidemia. Orphanet J Rare Dis 2014;9:130.

36. Wilcox G. Impact of pregnancy on inborn errors of metabolism. Rev Endocr Metab Disord 2018;19:13-33.

37. Vantyghem MC, Dobbelaere D, Mention K, et al. Endocrine manifestations related to inherited metabolic diseases in adults. Orphanet J Rare Dis 2012;7:11. 


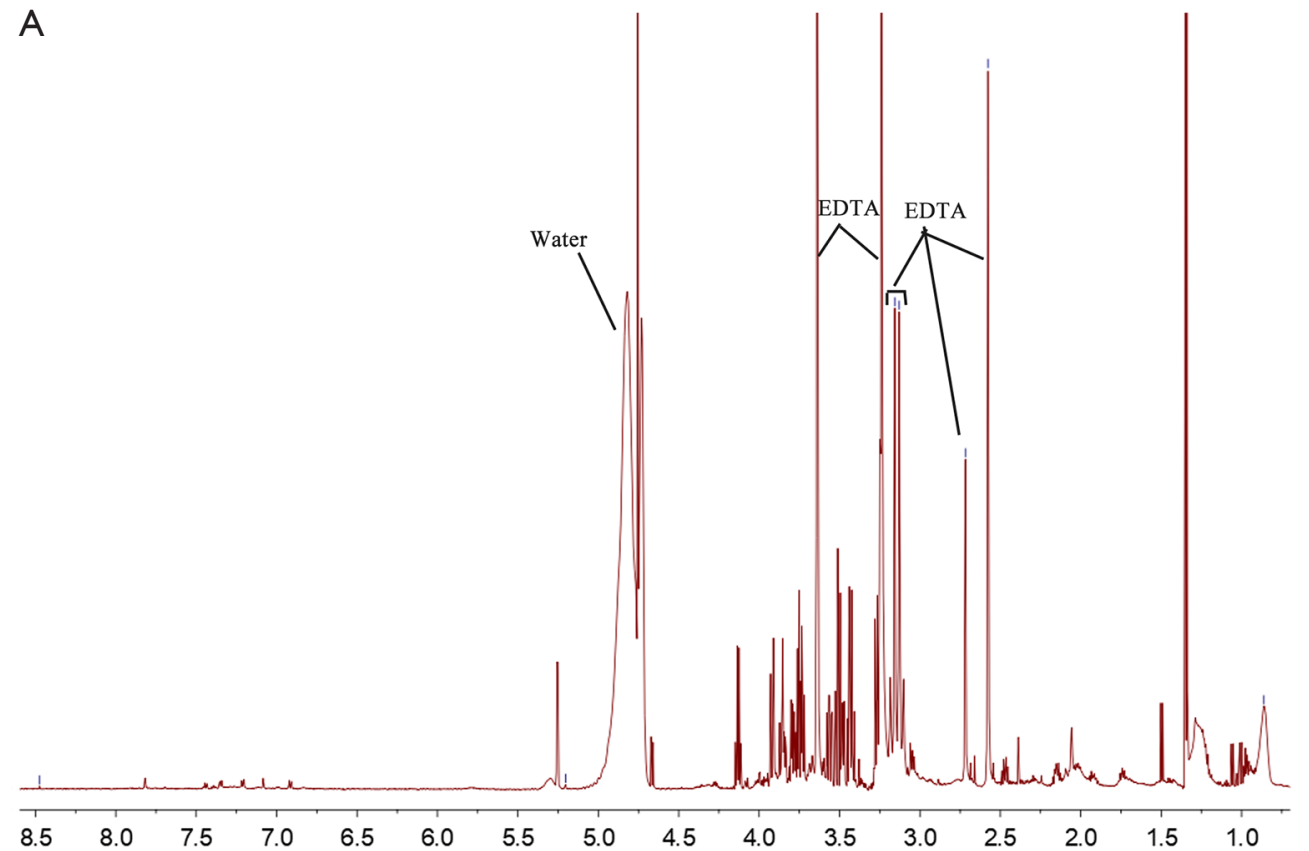

B

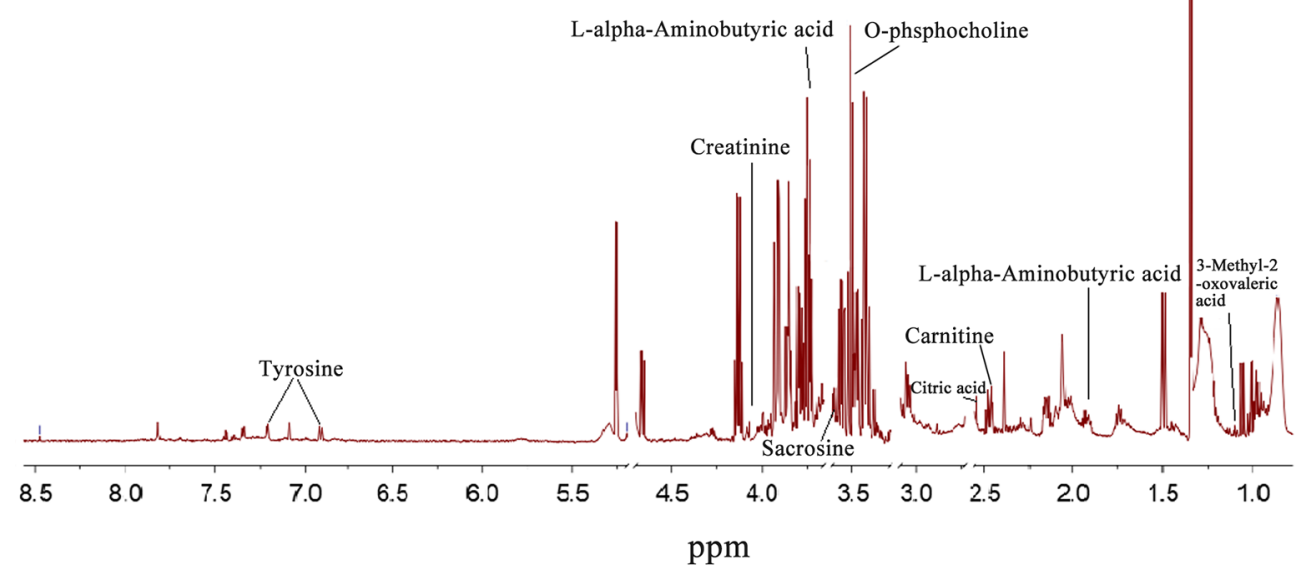

Figure S1 Sample 1H-NMR spectra obtained in the present study. (A) Original spectrum with the peaks of water and EDTA (as anticoagulant of the cord blood); (B) spectrum with deletion of the peaks of water and EDTA. The assigned metabolites were labeled. 

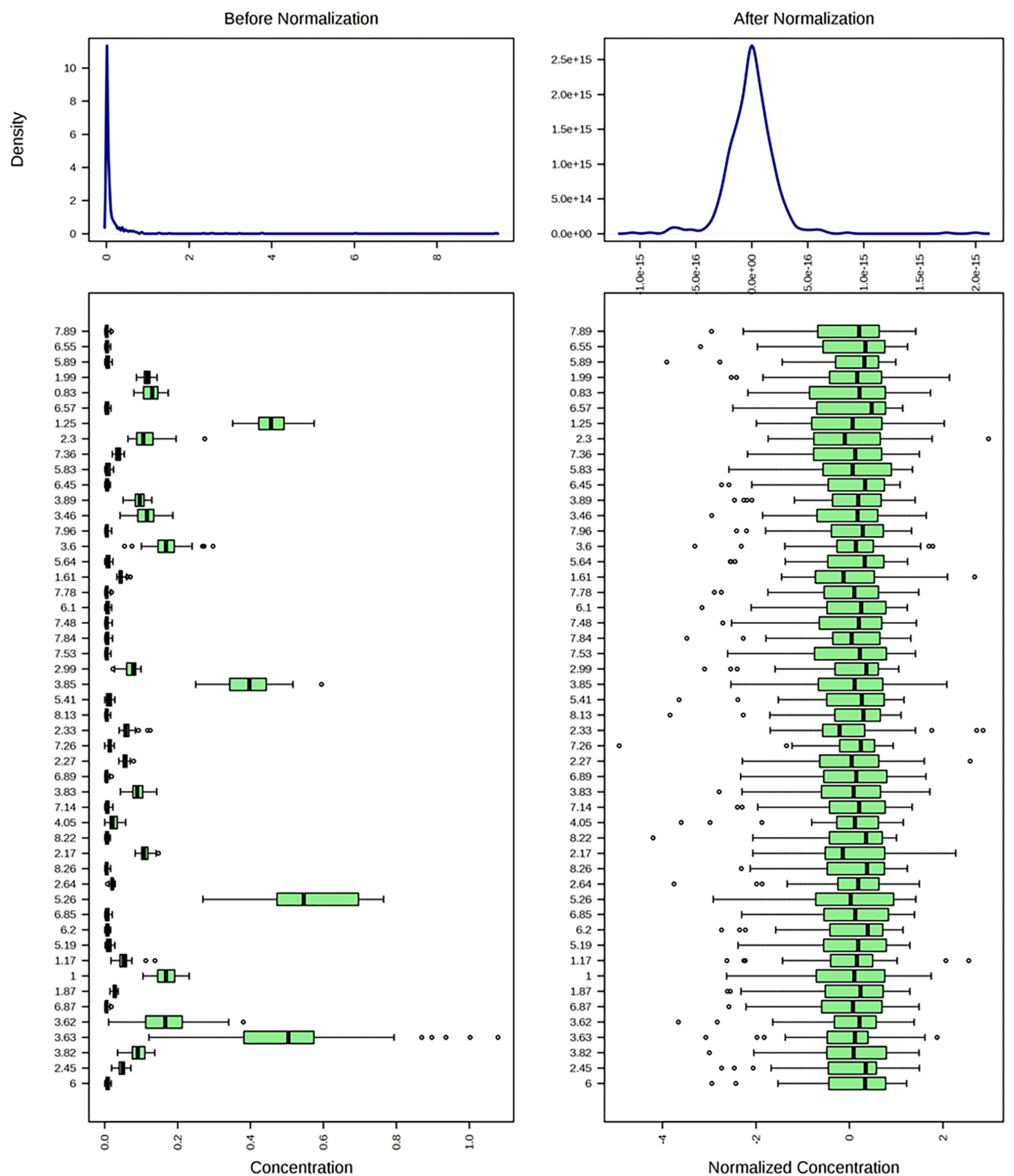

Figure S2 Normalization of the metabolomic data. The data normalization was carried out by the normalization module of MetaboAnalyst web portal. The normalization procedures are grouped into three categories: sample normalization (normalized by sum), data transformation (log transformation), data scaling (mean-centered and divided by the standard deviation of each variable). 


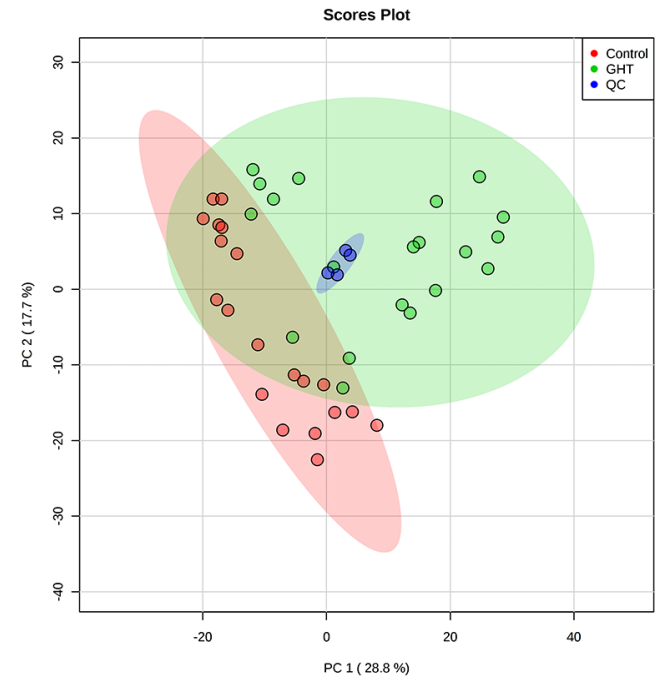

Figure S3 PCA score plot-based quality control of the metabolomic experiment. The 1H-NMR data of clinical samples and quality controls were analyzed by PCA. Red oval represents the 95\% CI of scores calculated from the NHT controls. Green oval represents the $95 \%$ CI of scores calculated from the GHT samples. Purple oval represents the 95\% CI of scores calculated from the quality controls. PCA, Principle Component Analysis; GHT, gestational hypothyroidism.

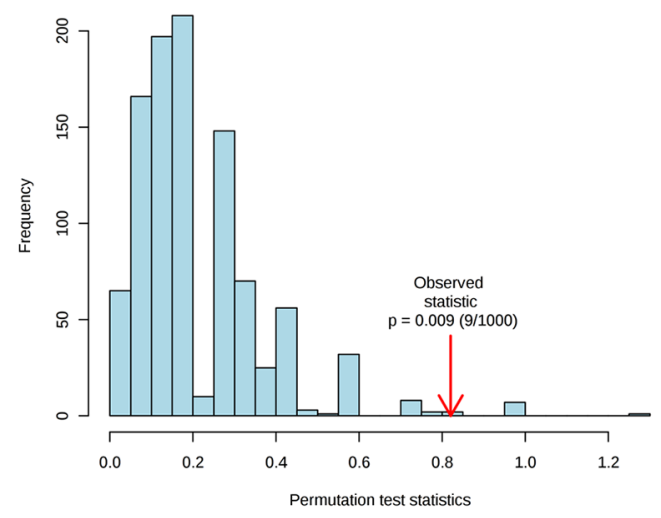

Figure S4 Permutation test of the PLS-DA model. The permutation test was carried out by the PLSDA permutation module of MetaboAnalyst web portal. Separation distance $(\mathrm{B} / \mathrm{W})$ was selected for statistic test, and the permutation number was set to 1,000 . An empirical P value of 0.009 was observed. PLS-DA, Projection to Latent Structures-Discrimination Analysis. 
Table S1 Differential metabolites between GHT group and the healthy control group by Wilcoxon Rank Test

\begin{tabular}{lccc}
\hline Metabolite & Chemical shift & P value & Adjusted P value \\
\hline L-alpha-Aminobutyric acid & 1.92 & $1.65 \mathrm{E}-06$ & $3.89 \mathrm{E}-05$ \\
Glutamine & 2.15 & $3.53 \mathrm{E}-03$ & $8.96 \mathrm{E}-03$ \\
Carnitine & 2.47 & $1.16 \mathrm{E}-03$ & $3.66 \mathrm{E}-03$ \\
Citric acid & 2.55 & $1.37 \mathrm{E}-06$ & $3.33 \mathrm{E}-05$ \\
Sarcosine & 2.71 & $8.36 \mathrm{E}-04$ & $2.91 \mathrm{E}-03$ \\
3-Methyl-2-oxovaleric acid & 2.94 & $8.34 \mathrm{E}-08$ & $5.69 \mathrm{E}-06$ \\
Tyrosine & 3.05 & $1.33 \mathrm{E}-08$ & $5.37 \mathrm{E}-06$ \\
Ethanolamine & 3.13 & $2.91 \mathrm{E}-03$ & $7.62 \mathrm{E}-03$ \\
Choline & 3.19 & $3.89 \mathrm{E}-03$ & $9.74 \mathrm{E}-03$ \\
Phosphoethanolamine & 3.23 & $7.47 \mathrm{E}-04$ & $2.65 \mathrm{E}-03$ \\
Glycine & 3.58 & $1.16 \mathrm{E}-03$ & $3.66 \mathrm{E}-03$ \\
Creatinine & 4.06 & $5.21 \mathrm{E}-05$ & $4.13 \mathrm{E}-04$ \\
O-phsphocholine & 4.16 & $2.58 \mathrm{E}-04$ & $1.21 \mathrm{E}-03$ \\
\hline
\end{tabular}

GHT, gestational hypothyroidism.

Table S2 Differential metabolites between GHT group and the healthy control group by EBAM

\begin{tabular}{|c|c|c|c|c|}
\hline Metabolite & Chemical shift & z.value & Posterior & local.fdr \\
\hline Glutamine & 2.15 & -3.59 & 0.99 & $6.02 \mathrm{E}-03$ \\
\hline Carnitine & 2.47 & -3.90 & 1.00 & $2.52 \mathrm{E}-03$ \\
\hline Citric acid & 2.55 & -5.68 & 1.00 & 2.04E-05 \\
\hline 3-Methyl-2-oxovaleric acid & 2.94 & -4.90 & 1.00 & 1.65E-04 \\
\hline Tyrosine & 3.05 & -7.26 & 1.00 & $3.25 \mathrm{E}-07$ \\
\hline Ethanolamine & 3.13 & -3.26 & 0.98 & $1.52 \mathrm{E}-02$ \\
\hline Choline & 3.19 & -2.85 & 0.95 & 4.64E-02 \\
\hline Creatinine & 4.06 & 4.55 & 0.99 & 5.87E-03 \\
\hline O-phosphocholine & 4.16 & 3.96 & 0.99 & 8.12E-03 \\
\hline
\end{tabular}

GHT, gestational hypothyroidism. 
Table S3 Clinical indices of the enrolled pregnant women in this study

\begin{tabular}{|c|c|c|c|c|c|c|c|c|c|}
\hline Sample & Group & DeltaBMI & $\begin{array}{l}\text { Fundal } \\
\text { height }\end{array}$ & $\begin{array}{c}\text { Abdomen } \\
\text { circumference }\end{array}$ & $\mathrm{TSH}^{\star}(\mathrm{mlU} / \mathrm{mL})$ & $\mathrm{FT}^{*}(\mathrm{pmol} / \mathrm{L})$ & $\mathrm{FT}^{*}(\mathrm{pmol} / \mathrm{L})$ & Type of delivery\# & $\begin{array}{c}\text { Gestational } \\
\text { age\# }\end{array}$ \\
\hline C.G55 & Control & 9.38 & 37 & 115 & 2.27 & 3.87 & 13.32 & Vaginal & $40+1$ \\
\hline C.G62 & Control & 7.72 & 34 & 96 & 1.69 & 5.89 & 18.5 & Vaginal & $39+1$ \\
\hline C.G92 & Control & 8.32 & 34 & 98 & 3.21 & 5.02 & 15.78 & Vaginal & 41 \\
\hline C.J19 & Control & 4.25 & 30 & 98 & 3.31 & 3.67 & 15.21 & Vaginal & $37+2$ \\
\hline C.J66 & Control & 5.27 & 31 & 101 & 1.53 & 4.84 & 14.59 & Vaginal & $39+2$ \\
\hline C.J75 & Control & 2.54 & 34 & 95 & 2.7 & 4.45 & 12.68 & Vaginal & $37+3$ \\
\hline C.J79 & Control & 4.24 & 31 & 96 & 2.5 & 3.4 & 12.26 & Vaginal & $39+3$ \\
\hline C.J86 & Control & 3.80 & 33 & 96 & 3.56 & 4.51 & 15.79 & Vaginal & $37+2$ \\
\hline C.J98 & Control & 4.25 & 32 & 93 & 1.37 & 3.97 & 16.21 & Vaginal & $38+4$ \\
\hline C.T129 & Control & 8.76 & 33 & 128 & 3.19 & 5.15 & 14.54 & Vaginal & $40+1$ \\
\hline С.T26 & Control & 5.14 & 34 & 100 & 1.3 & 4.03 & 15.06 & Vaginal & $40+2$ \\
\hline С.T29 & Control & 2.48 & 35 & 106 & 1.73 & 4.87 & 17.29 & Vaginal & $39+2$ \\
\hline С.T32 & Control & 4.01 & 30 & 88 & 1.12 & 5.63 & 19.32 & Vaginal & 40 \\
\hline C.T34 & Control & 7.09 & 34 & 110 & 1.06 & 5.03 & 16.31 & Vaginal & 41 \\
\hline C.T45 & Control & 7.62 & 35 & 100 & 1.03 & 3.29 & 12.63 & Vaginal & $40+2$ \\
\hline C.T8 & Control & 1.08 & 33 & 107 & 2.31 & 3.91 & 16.05 & Vaginal & $40+1$ \\
\hline C.Z32 & Control & 4.78 & 35 & 91 & 2.64 & 4.08 & 13.32 & Vaginal & $40+2$ \\
\hline C.Z73 & Control & 5.86 & 35 & 102 & 3.58 & 2.19 & 10.53 & Vaginal & $40+2$ \\
\hline JJ.D103 & GHT & 3.91 & 34 & 91 & 3.69 & 34.24 & 14.07 & Vaginal & $39+2$ \\
\hline JJ.D150 & GHT & 10.65 & 34 & 110 & 7.18 & 5.41 & 11.97 & Vaginal & $40+2$ \\
\hline JJ.G39 & GHT & 6.02 & 35 & 109 & 4.84 & 3.69 & 14.57 & Vaginal & 41 \\
\hline JJ.G58 & GHT & 3.01 & 35 & 95 & 6.85 & 4.88 & 15.12 & Vaginal & $38+4$ \\
\hline JJ.G64 & $\mathrm{GHT}$ & 4.25 & 32 & 93 & 5.54 & 5.77 & 17.32 & Vaginal & 39 \\
\hline JJ.G96 & $\mathrm{GHT}$ & 6.17 & 32 & 101 & 4.42 & 4.9 & 15.98 & Vaginal & $38+4$ \\
\hline JJ.J25 & $\mathrm{GHT}$ & 6.81 & 35 & 103 & 5.77 & 4.84 & 13.86 & Vaginal & $40+3$ \\
\hline JJ.J58 & $\mathrm{GHT}$ & 5.51 & 35 & 105 & 6.8 & 5.4 & 14.53 & Vaginal & 40 \\
\hline JJ.T149 & GHT & 7.35 & 33 & 115 & 5.52 & 5.52 & 5.02 & Vaginal & $39+1$ \\
\hline JJ.T18 & $\mathrm{GHT}$ & 6.25 & 33 & 113 & 11.13 & 4.44 & 12.16 & Vaginal & $39+2$ \\
\hline JJ.T51 & $\mathrm{GHT}$ & 3.81 & 33 & 96 & 4.92 & 3.66 & 13.95 & Vaginal & 39 \\
\hline JJ.T73 & $\mathrm{GHT}$ & 8.25 & 33 & 98 & 7.85 & 6.22 & 15.14 & Vaginal & $38+2$ \\
\hline JJ.Z59 & $\mathrm{GHT}$ & 5.86 & 34 & 102 & 4.68 & 5.2 & 12.8 & Vaginal & $39+1$ \\
\hline JJ.Z60 & $\mathrm{GHT}$ & 5.38 & 34 & 103 & 4.21 & 5.09 & 13.09 & Vaginal & $40+1$ \\
\hline JJ.Z75 & GHT & 3.65 & 33 & 101 & 4.76 & 3.41 & 11.24 & Vaginal & $40+1$ \\
\hline JJ.Z83 & $\mathrm{GHT}$ & 4.87 & 33 & 97 & 8.53 & 4.79 & 12.11 & Vaginal & $39+3$ \\
\hline JJ.Z87 & GHT & 8.45 & 34 & 102 & 4.85 & 4.8 & 15.69 & Vaginal & $39+3$ \\
\hline JJ.Z99 & GHT & 7.23 & 34 & 104 & 4.63 & 5.31 & 15.73 & Vaginal & $38+2$ \\
\hline
\end{tabular}

*, measured at 11th-14th gestational week/after the parturition; \#, Data collected after the childbirth. DeltaBMl, the difference between $\mathrm{BMI}$ at 11th-14th gestational week and BMI before pregnancy; TSH, thyroid-stimulating hormone; FT3, free tri-iodothyonine; FT4, free thyroxine. 